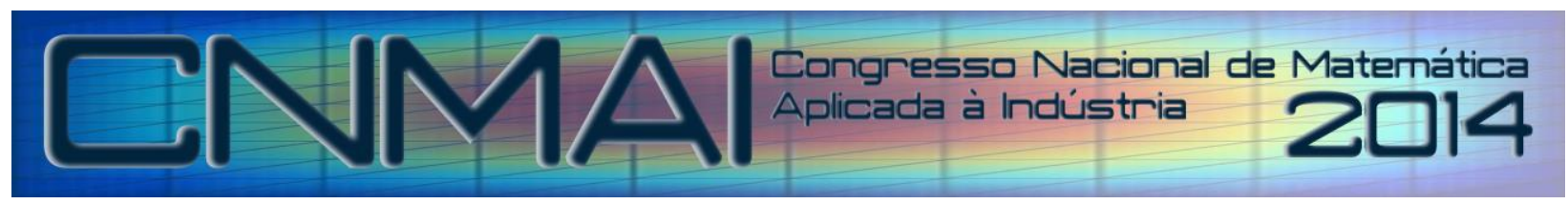

18 a 21 de novembro de 2014, Caldas Novas - Goiás

\title{
CNMAI2014-0088 - ESTUDO COMPARATIVO DA CONVERGÊNCIA DE MÉTODOS NUMÉRICOS ITERATIVOS APLICADOS NA SOLUÇÃO DA EQUAÇÃO DE TRANSFERÊNCIA DE CALOR PROPOSTA POR ROSENTHAL SOB A VARIAÇÃO DE PARÂMETROS DE SOLDAGEM.
}

\author{
Antonio do Nascimento Silva Alves, ansa@usp.br ${ }^{1}$ \\ Rubelmar Maia de Azevedo Cruz Neto, rubelmar.neto@usp.br ${ }^{1}$ \\ Sergio Duarte Brandi, sebrandi@usp.br ${ }^{1}$ \\ Dario Magno Batista Ferreira, dariomagno@usp.br ${ }^{1}$
}

${ }^{1}$ USP - Dep. de Eng. Metalúrgica e de Materiais. Av. Professor Mello Moraes, 2463, CEP 05508-030, São Paulo - SP

\begin{abstract}
Resumo: A obtenção de raizes ou zero de função de equações transcendentais na forma reduzida, depende do comportamento destas equações próximo à essas raizes, necessitando assim de métodos numéricos confiáveis e de baixo custo computacional para sua determinação mais eficaz, métodos estes amplamente utilizados nas áreas de engenharia. A geometria das isotermas em juntas soldadas de chapas metálicas são de fundamental importancia para determinação das propriedades mecânicas, formação da microestrutura e taxa de resfriamento, para isso, utiliza-se a solução da equação de transferência de calor proposta por Rosenthal, considerando usualmente a condução como modo de transferência de calor, desconsiderando as contribuições da convecção e radiação. Ao variar os parametros de soldagem, a saber, corrente, tensão, velocidade de soldagem e eficiência de processo, tem-se como consequência, diferentes geometrias das isotermas. Para solução da equação supra citada, utiliza-se métodos numéricos iterativos. O presente trabalho apresenta um estudo comparativo da estabilidade de convergência dos métodos da Bisseção, Newton-Raphson e Secante em termos do número de iterações, tempo computacional e precisão do erro numérico na obtenção das raizes ou zero de função. Sob estas condições, o que apresentou melhor convergência foi o de Newton-Raphson com um número menor de iterações, em um menor tempo computacional quando comparado aos outros métodos.
\end{abstract}

Palavras-chave: Transferência de calor na soldagem, Métodos Numéricos, Contorno de Isotermas, Tempo computacional, Equação de Rosenthal.

\section{INTRODUÇÃO}

A soldagem é um dos processos de fabricação mais importantes da indústria, sendo amplamente utilizado na fabricação de estruturas metálicas, navios, oleodutos, plataformas marítimas, etc. Os processos de soldagem utilizam o calor como principal fonte de energia, sendo necessário o seu fornecimento à junta, para a formação da poça de fusão, de tal maneira a garantir a execução de uma junta soldada de boa qualidade. Este calor é de suma importância na execução da junta soldada, no entanto, possui grande potencial de problemas devido a sua influência direta nas transformações de fase que afetam o desempenho da junta soldada, Wainer, Brandi e Mello (1992).

Esses efeitos são consequência dos ciclos térmicos e, consequentemente, das variações de temperatura em diversos pontos do material a ser soldado. Por utilizar uma fonte de calor concentrada com geometria puntiforme (chapa grossa) ou linear (chapa fina), a soldagem é caracterizada pelo aquecimento de regiões localizadas na peça, permanencendo o restante desta em temperaturas muito inferiores, denotando a não uniformidade da distribuição de temperatura na junta soldada, Guimarães et al., (2012).

A determinação da geometria do contorno das isotermas provenientes das juntas soldadas em chapas metálicas é fundamental para o entendimento do comportamento térmico do processo para diferentes materiais e parâmetros de soldagem. Esse contorno da isoterma é obtido através da solução da equação de transferência de calor por condução, proposta por Rosenthal em 1941, na forma reduzida. Através da solução desta equação é possivel determinar os pontos 
que compõem o contorno da isoterma, para uma determinada temperatura, admitindo cada ponto como a raiz da equação de Rosenthal na forma reduzida ao longo do eixo das ordenadas em relação ao das abscissas.

Para esse fim, foram utilizados métodos numéricos iterativos para obtenção das raízes, tais como: Bisseção, Newton- Raphson e Secante. Estes métodos possuem, em termos gerais, uma grande aplicação na solução de diferentes problemas de engenharia.

\section{MODELO MATEMÁTICO}

\subsection{Modelo de Chapa Grossa (fonte de calor com geometria puntiforme)}

De acordo com a Fig. 1, o modelo geral de chapa grossa consiste em um corpo isotrópico semi-infinito, com temperatura inicial $\left(T_{0}\right)$ e fonte de calor com o formato puntiforme. As propriedades térmicas são assumidas como constantes e independentes da temperatura, bem como as perdas de calor por convecção e radiação são desprezadas.

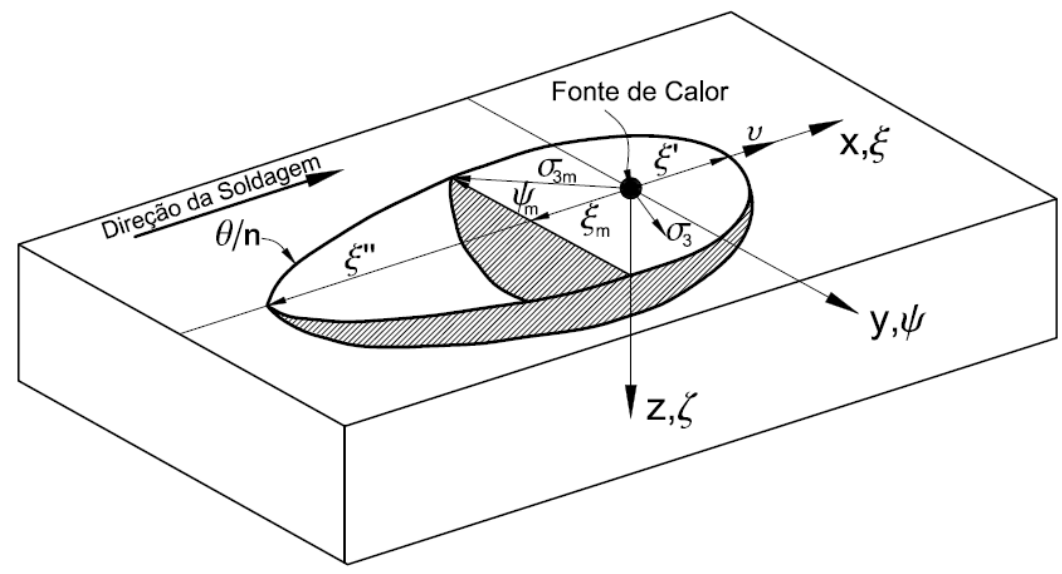

Figura 1. Representação gráfica tridimensional da solução de chapa grossa de Rosenthal (esquemático), adaptado de Myhr and Grong ( 1990).

A partir da análise de Rosenthal (1941), assumindo tais condições, e admitindo que a condução de calor ocorre nas três direções, a distribuição de calor na chapa no regime quase estacionário é dada pela Eq. 1, Grong (1997).

$$
T-T_{0}=\frac{q_{0}}{2 \pi \lambda} \frac{1}{R_{3}} \exp \left[-\frac{\sigma}{2 \alpha}\left(R_{3}+x\right)\right]
$$

Onde :

$$
R_{3}=\sqrt{x^{2}+y^{2}+z^{2}}
$$

As Eqs. (1a) e (1b) podem ser escritas de forma mais generalizada, utilizando grupos adimensionais apropriados, como mostrados a seguir, onde $\theta$ é a temperatura adimensional $\left(T_{m}\right), n$ é chamado parâmetro operacional, $\xi, \psi$ e $\delta$ são as coordenadas adimensionais e $\phi$, o vetor raio.

Temperatura adimensional:

$$
\theta=\frac{\left(T-T_{0}\right)}{\left(T_{m}-T_{0}\right)}
$$

Parâmetro Operacional:

$$
n=\frac{q_{0} v}{4 \pi a^{2} \rho c\left(T_{m}-T_{0}\right)}=\frac{q_{0} v}{4 \pi a^{2}\left(H_{m}-H_{0}\right)}
$$


Onde:

$q_{0}$ É a potência do $\operatorname{arco}(\mathrm{W}), v$ é a velocidade de soldagem $(\mathrm{mm} / \mathrm{s}), a$ a difusividade térmica $\left(\mathrm{mm}^{2} / \mathrm{s}\right)$, e $\rho c$ o calor específico volumétrico $\left(\mathrm{J} /{ }^{\circ} \mathrm{C} \mathrm{mm}{ }^{3}\right)$.

Coordenada $x$ adimensional:

$$
\xi=\frac{v}{2 a} x
$$

Coordenada $y$ adimensional:

$$
\psi=\frac{v}{2 a} y
$$

Coordenada $z$ adimensional:

$$
\phi=\frac{v}{2 a} z
$$

Espessura da chapa $d$ adimensional:

$$
\delta=\frac{v}{2 a} d
$$

Substituindo os parâmetros adimensionais acima na Eq. (1a) e (1b), nos obtemos:

$$
\frac{\theta}{n}=\frac{1}{\sigma_{3}} \exp \left(-\sigma_{3}-\xi\right)
$$

Onde,

$$
\sigma_{3}=\sqrt{\xi^{2}+\psi^{2}+\phi^{2}}
$$

Aplicando a diferenciação parcial na Eq. (3a), obtém-se o termo $\xi_{m}$, como mostrado a seguir.

$$
\begin{gathered}
\frac{\partial(\ln \theta / n)}{\partial \sigma_{3}}=-\frac{1}{\sigma_{3 m}}-1-1 \cdot \frac{\sigma_{3 m}}{\xi_{m}}=0 \\
\xi_{m}=-\frac{-\left(\sigma_{3 m}\right)^{2}}{\sigma_{3 m}+1}
\end{gathered}
$$

Substituindo-se a Eq. (4b) na Eq. (3a) obtém-se:

$$
\frac{\theta}{n}=\frac{1}{\sigma_{3 m}} \exp \left[-\frac{\sigma_{3 m}}{\left(\sigma_{3 m}+1\right)}\right]
$$

A Eq. (4c) pode ser utilizada para determinar o $\psi_{m}$, que é a altura máxima do contorno da isoterma como mostrado na Fig. (1), e dado por:

$$
\psi_{m}=\sqrt{\left(\sigma_{3 m}\right)^{2}-\left(\xi_{m}\right)^{2}}=\frac{\sigma_{3 m}}{\left(\sigma_{3 m}+1\right)} \sqrt{1+2 \sigma_{3 m}}
$$

O comprimento total do invólucro da isoterma pode ser obtido a partir de $\xi_{t}=\xi^{\prime}-\xi^{\prime \prime}$, onde o $\xi^{\prime}$ e o $\xi^{\prime \prime}$ são as distâncias tomadas como referência em relação à posição da fonte de calor. As coordenadas $\xi^{\prime} \mathrm{e} \xi^{\prime \prime}$ são obtidas pela manipulação de $\sigma_{3}= \pm \xi$ na Eq.(3a), como mostrado a seguir: 


$$
\begin{gathered}
\xi^{\prime}=\frac{1}{2} \ln \left(\frac{n / \theta}{\xi^{\prime}}\right) \\
\xi^{\prime \prime}=\frac{-n}{\theta}
\end{gathered}
$$

\subsection{Modelo de Chapa Intermediária}

O modelo de chapa intermediária apresentado por Rosenthal (1941) considera a fonte de calor puntiforme movendo-se com velocidade constante ao longo da chapa, admite ainda que a perda de calor na superficie da chapa para vizinhança é igual a zero. Diante dessas considerações, admite-se a reflexão espelhada de fontes imaginárias, acima de $\mathrm{z}=0$ e abaixo de $\mathrm{z}=\mathrm{d}$, como apresentado esquematicamente na Fig(2), conhecido como método das imagens.

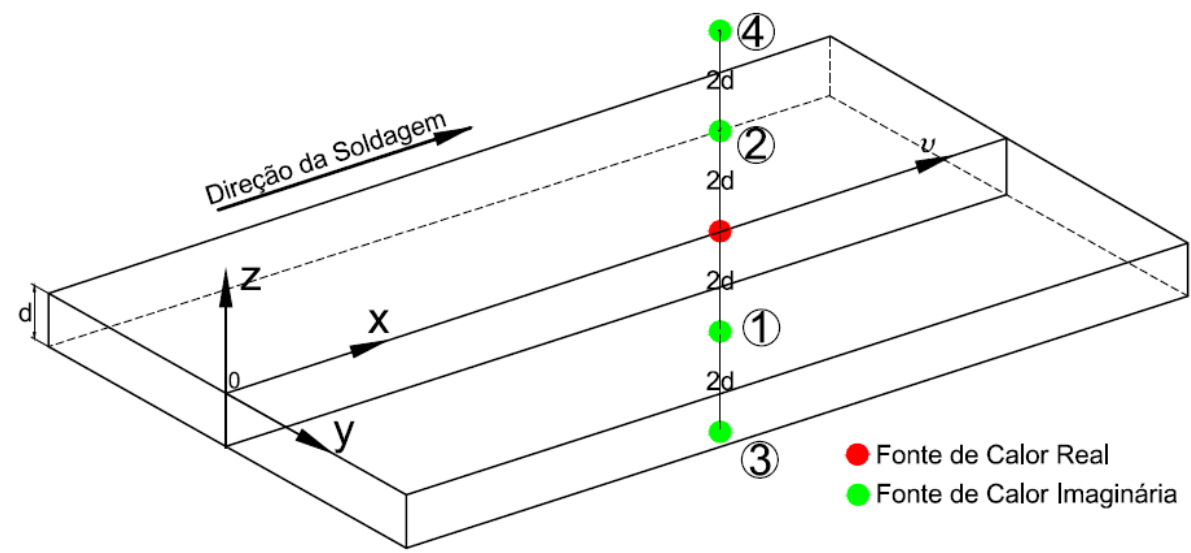

Figura 2. Fonte real e fontes imaginárias espelhadas simetricamente para modelagem da condição de chapa intermediária.

As fontes imaginárias são posicionadas simetricamente em distâncias $\pm 2 i d$ acima e abaixo da superfície da chapa, a distribuição de temperatura no regime quase estacionário pode ser obtido na forma de uma série convergente como mostra a Eq.(8a).

$$
T-T_{0}=\frac{q_{0}}{2 \pi \lambda} \exp \left(-\frac{v x}{2 \alpha}\right) \cdot\left[\sum_{i=-\infty}^{i=+\infty} \frac{1}{R i} \exp \left(-\frac{v}{2 \alpha} R i\right)\right]
$$

Onde :

$$
R_{i}=\sqrt{x^{2}+y^{2}+(z-2 i d)^{2}}
$$

Diante disto nota-se que a Eq. (8a) é simplesmente a solução geral para chapa grossa de Rosenthal (1941), somando cada fonte de calor, ou seja a Eq. (8a) próxima ao centro da soldagem, torna-se a Eq.(1a) Grong (1997).

Substituindo os parâmetros adimensionais definidos acima na Eq.(8a) temos:

$$
\frac{\theta}{n}=\exp (-\xi)\left[\sum_{i=-\infty}^{i=+\infty}\left(\frac{1}{\sigma_{i}}\right) \exp \left(-\sigma_{i}\right)\right]
$$

Onde:

$$
\sigma_{i}=\frac{v}{2 a} R_{i}=\sqrt{\xi^{2}+\psi^{2}+(\zeta-2 i \delta)^{2}}
$$


Neste trabalho será utilizado o modelo de chapa intermediária, a fim de avaliar o comportamento dos métodos numéricos diante das variações de propriedades térmicas dos materiais e dos parâmetros de soldagem, como mostrado na Tab. (1).

\section{MATERIAIS E MÉTODOS}

A geometria da isoterma entre outros fatores é determinada pela velocidade de soldagem e o equilíbrio entre o aporte de calor e as condições de resfriamento da peça, equilíbrio esse, que é dependente das propriedades térmicas do material da chapa metálica. Durante o deslocamento da fonte de calor, os contornos das isotermas continuam constantes indicando que o estado quase estacionário foi alcançado, isto é, $\partial T / d t=0$.

O parâmetro operacional adimensional $n$, Eq. (2b) possui influência direta na geometria da isoterma. A Fig.(3a) mostra um contorno que se aproxima de uma circunferência, com um baixo valor de $q_{0}$ e $v$, consequentemente, um $n$ baixo. Por outro lado, a Fig.(3b) apresenta um contorno mais alongado aproximando-se de um formato de "gota", favorecido pelo $n$ elevado, que é resultando do aumento a velocidade de soldagem. No entanto é importante notar que as propriedades térmicas do metal de base são importantes, desde que $n$ esteja em função de $\alpha$ e $\left(H_{m}-H_{0}\right)$. Consequentemente, o formato alongado (gota) é usualmente observado em soldagem com baixa difusividade térmica, enquanto que o contorno em formato esférico é mais suscetível de surgir durante a soldagem de alumínio, devido à alta difusividade térmica deste material.
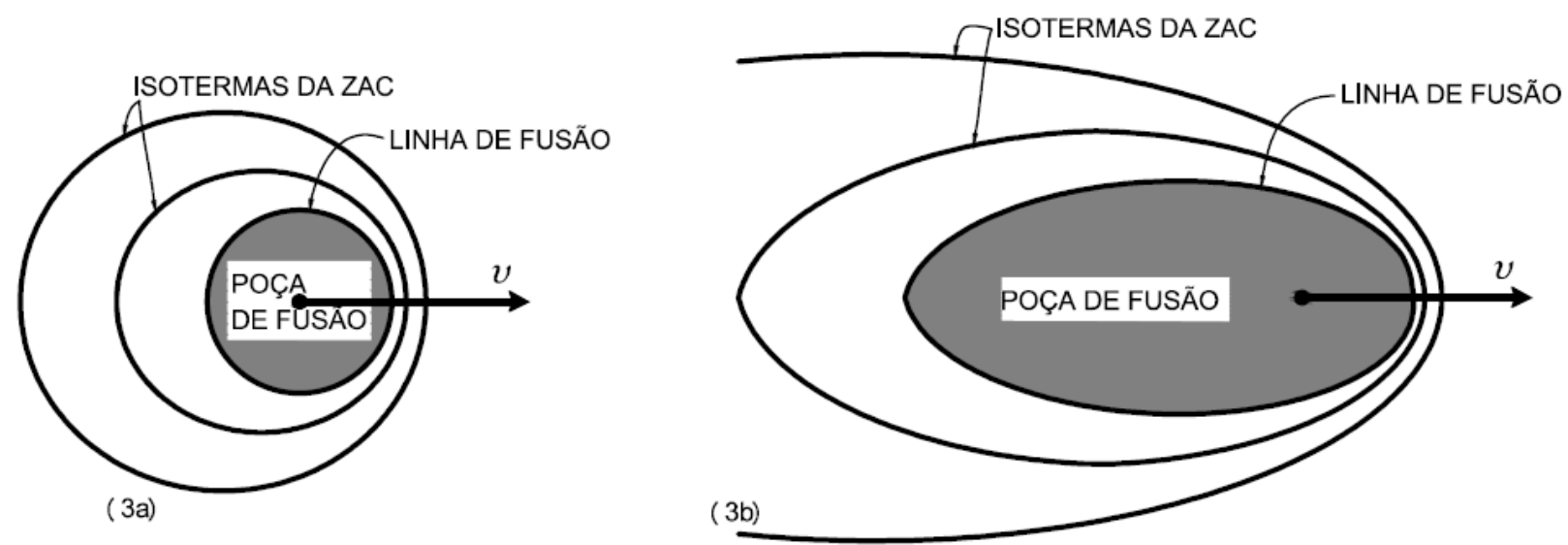

Figura 3. Formato teórico do contorno da linha de fusão e as isotermas vizinhas sob diferentes condições: (3a) baixo valor de $n$, (3b) alto valor de $n$, adaptado de Grong (1997).

A geometria da isoterma pode demonstrar fortemente a mudança na formação da microestrutura e suas implicações nas propriedades mecânicas do metal de solda e da zona afetada pelo calor. Em um contorno próximo do formato de circunferência, a zona fundida apresentará grãos colunares que se entrelaçam no centro do cordão, devido ao deslocamento no sentido do gradiente térmico máximo a partir da linha de fusão. Por outro lado, o formato alongado (gota) forma grãos colunares que podem se encontrar no centro do cordão, formando um plano preferencial, , o que acarreta no surgimento de uma trinca na linha de centro da poça de fusão, Grong (1997).

O problema simulado neste trabalho, tem como base o estudo de caso apresentado por Grong (1997), no Capítulo 1 (Heat Flow and Temperature Distribution Welding), considerando o processo GMAW em duas chapas com espessura de $12,5 \mathrm{~mm}$ de acordo com parâmetros e propriedades apresentados na Tab. 1.

Tabela 1. Parâmetros operacionais e propriedades térmicas do material a ser soldado.

\begin{tabular}{|c|c|c|c|c|c|c|c|c|c|}
\hline \multirow[b]{2}{*}{ Casos } & \multicolumn{5}{|c|}{ Parâmetros operacionais } & \multirow[b]{2}{*}{ Material } & \multicolumn{3}{|c|}{ Propriedades Térmicas do materia } \\
\hline & $\begin{array}{c}\text { Corrente } \\
\text { (A) }\end{array}$ & $\begin{array}{c}\text { Tensão } \\
\text { (V) }\end{array}$ & $\eta$ & $\begin{array}{c}v \\
(\mathrm{~mm} / \mathrm{s})\end{array}$ & $\begin{array}{c}\mathrm{T}_{0} \\
\left({ }^{\circ} \mathrm{C}\right)\end{array}$ & & $\begin{array}{c}\mathrm{T}_{\mathrm{m}} \\
\left({ }^{\circ} \mathrm{C}\right)\end{array}$ & $\begin{array}{c}\rho c \\
\left(\mathrm{~J} / \mathrm{mm}^{3 \circ} \mathrm{C}\right)\end{array}$ & $\begin{array}{c}\alpha \\
\left(\mathrm{mm}^{2} / \mathrm{s}\right)\end{array}$ \\
\hline Caso A1 & 150 & 25 & 0.8 & 2.5 & 20 & \multirow{2}{*}{ Liga de Al-Mg-S } & 652 & 0.0027 & 62 \\
\hline Caso A2 & 300 & 25 & 0.8 & 5.0 & 20 & & 652 & 0.0027 & 62 \\
\hline Caso B1 & 240 & 25 & 0.8 & 4.0 & 20 & \multirow{2}{*}{ Aço baixa liga } & 1520 & 0.005 & 5 \\
\hline Caso B2 & 480 & 25 & 0.8 & 8.0 & 20 & & 1520 & 0.005 & 5 \\
\hline
\end{tabular}




\subsection{Métodos Numéricos.}

Para avaliar este estudo de caso foram aplicados três métodos numéricos iterativos: Bisseção, Newton-Raphson e Secante. No método da Bisseção toma-se uma função $f(x)=0$, contínua em um intervalo fechado, $[a, b]$, de tal modo que $f(a) . f(b)<0$. Em seguida, a função $f(x)=0$ possui pelo menos uma raiz ou zero de função neste intervalo $[a, b]$. O método requer a redução pela metade dos subintervalos de $[a, b]$ repetidas vezes, Ehiwario (2014).

O método de Newton-Raphson encontra a inclinação da função (linha tangente) no ponto atual e utiliza o zero da linha tangente que intercepta o eixo $x$ como o próximo ponto desejado. O processo é repetido até que a raiz seja encontrada. Este método é provavelmente o mais popular utilizado para solução de equações não lineares, no entanto requer o cálculo da derivada da função, o que nem sempre é trivial. Além disso, a linha tangente pode perder o controle e ficar presa em um "loop" e, consequentemente, não ocorrendo a convergência requerida. O processo de iteração de Newton-Raphson é dado por:

$$
x_{k+1}=x_{k}-\frac{f(x)}{f^{\prime}(x)}, k=0,1,2, \ldots
$$

Como foi dito anteriormente uma das implicações que restringe o uso do método de Newton-Raphson para alguns casos, é encontrar o valor da derivada da função a cada iteração. Existem algumas funções que são extremamente difíceis, se não impossíveis, a derivação ou computacionalmente dispendiosas. O método da secante é uma aproximação de Newton-Raphson, o que os diferencia é substituição da derivada por um quociente de diferença, da Eq. (10), para a aproximação da derivada temos que:

$$
f^{\prime}(x) \approx \frac{f\left(x_{k}\right)-f\left(x_{k-1}\right)}{x_{k}-x_{k-1}} \text {, onde } x_{k} \text { e } x_{k+1} \text { são duas aproximações para a raiz, a função de iteração para o }
$$

método da secante torna-se: $x_{k+1} \approx x_{k}-\frac{f\left(x_{k}\right)}{\frac{f\left(x_{k}\right)-f\left(x_{k-1}\right)}{x_{k}-x_{k-1}}}, x_{k+1} \approx x_{k}-\frac{f\left(x_{k}\right) \cdot\left(x_{k}-x_{k-1}\right)}{f\left(x_{k}\right)-f\left(x_{k-1}\right)}$,essa é a razão incremental do método da secante, Biswa (2011).

De acordo com Vetter, Santos e Marques (2006), o método da secante apresenta-se como uma alternativa ao método de Newthon-Raphson caso a função apresente dificuldade ao calcular a sua derivada. O critério de parada é muito importante na secante, pois se o objetivo for reduzir o intervalo que contém a raiz, apesar de atuar no intervalo, pode não atingir a precisão requerida. Nesse caso não é recomendado a utilização do método da secante. Considerando que o método ideal seria aquele em que a convergência estivesse assegurada, a ordem de convergência fosse alta e os cálculos por iterações fossem simples, o método de Newton-Raphson é o mais indicado sempre que for fácil verificar as condições de convergência, e que o cálculo da derivada não seja muito elaborado, conforme (Vetter, Santos e Marques ,2006; Cota, Bastos e Ferreira, 2012).

Miranda, et al. (2006), relata em seu trabalho que o processo de iteração é repetido até se obter a precisão estabelecida, ou seja, os três métodos numéricos são executados até que a condição de erro seja satisfeita, que é simplesmente uma diferença entre a leitura calculada e a obtida anteriormente. No caso das três rotinas, a precisão estabelecida foi de $10^{-5}$.

\subsection{Rotina Computacional.}

Elaborou-se um código computacional utilizando a plataforma Wolfram Mathematica $9.0^{\circledR}$ implementando os métodos numéricos para avaliação de desempenho, tendo como dados de entrada as informações contidas na Tab. 1.

Após determinar os limites da isoterma, $\xi^{\prime}$ e $\xi^{\prime \prime}$ o código inicia a obtenção dos pontos do contorno da isoterma utilizando a Eq. (8a) na forma reduzida, vale atentar que o código calcula apenas a isoterma na superfície da chapa, após realizar uma varredura discretizando o intervalo $\left[\xi^{\prime}, \xi^{\prime \prime}\right]$, como mostra a Fig. 1, ou seja, para cada ponto no eixo $x$ é determinado um ponto correspondente no eixo $y$, é gerada uma tabela com estes pontos e então interpola-se obtendo o contorno da isoterma. A configuração do computador utilizado na realização das simulações é:

- Processador: Intel(R) Core (TM) i7-2600 CPU @ 3.40GHz

- Memória Instalada (RAM): $4.00 \mathrm{~GB}$

- Tipo de Sistema: 32-bit 


\section{RESULTADOS E DISCUSSÕES}

As Figs. (4) e (5), apresentam os resultados da simulação para os casos A e B respectivamente, a partir dos parâmetros apresentados da Tab. (1), para temperaturas de acordo com o material do metal de base.

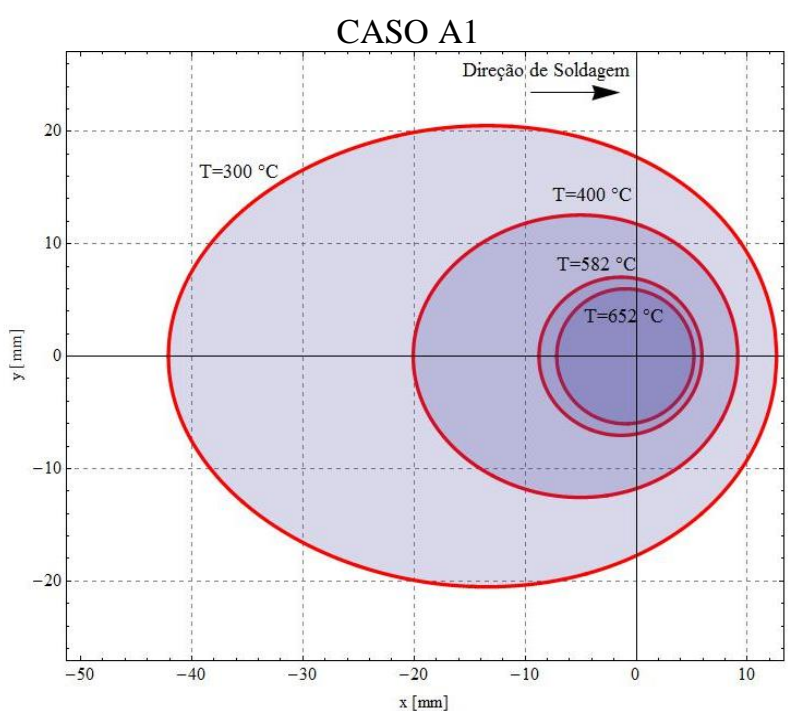

(a)

Figura (4a). Formatos dos contornos das isotermas do caso A1, Corrente $=150 \mathrm{~A}$ e a velocidade de soldagem $v=2.5 \mathrm{~mm} / \mathrm{s}$.

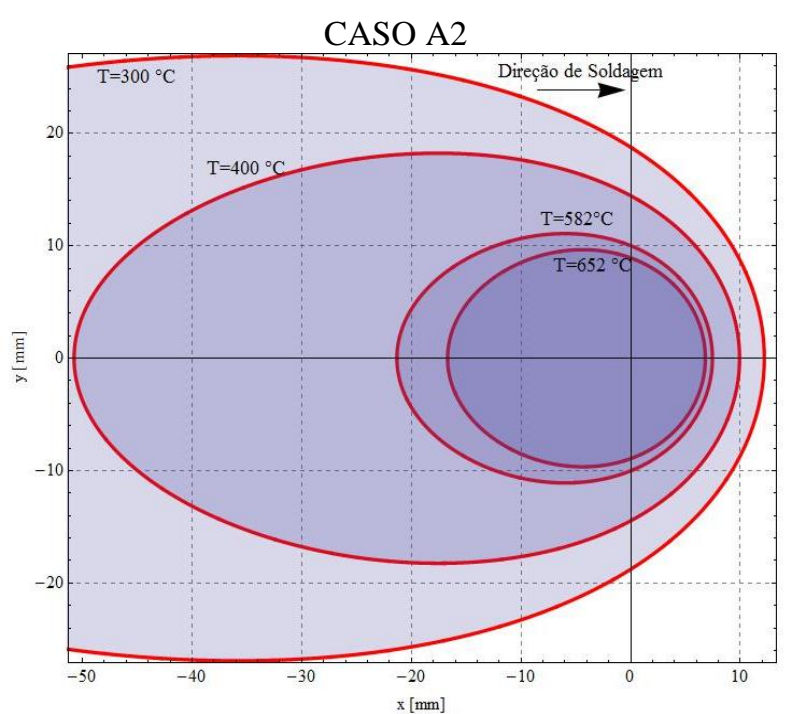

(b)

Figura (5b). Formatos dos contornos das isotermas do caso A2, Corrente $=300$ A e a velocidade de soldagem $v=5 \mathrm{~mm} / \mathrm{s}$.

Comparando-se a temperatura na linha de fusão ( temperatura de fusão do alumínio é $652{ }^{\circ} \mathrm{C}$ ) da Fig. (4a) e (4b), percebe-se a forte influência da velocidade de soldagem no formato do contorno desta isoterma. É evidente que o alongamento do contorno da isoterma da linha de fusão, e suas isotermas vizinhas, deve-se ao fato do aumento da velocidade de soldagem de $2,5 \mathrm{~mm} / \mathrm{s}$ para $5,0 \mathrm{~mm} / \mathrm{s}$ e como também o aumento da corrente de soldagem e, consequentemente, da potência do arco.

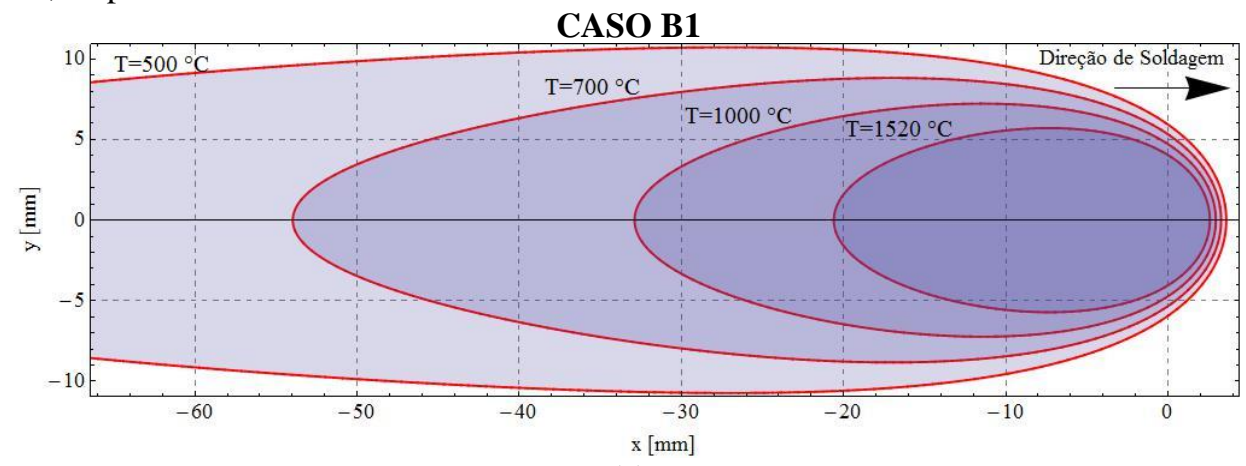

(a)

Figura (5a). Formatos dos contornos das isotermas do caso B1, Corrente $=240$ A e a velocidade de soldagem $v=4 \mathrm{~mm} / \mathrm{s}$.

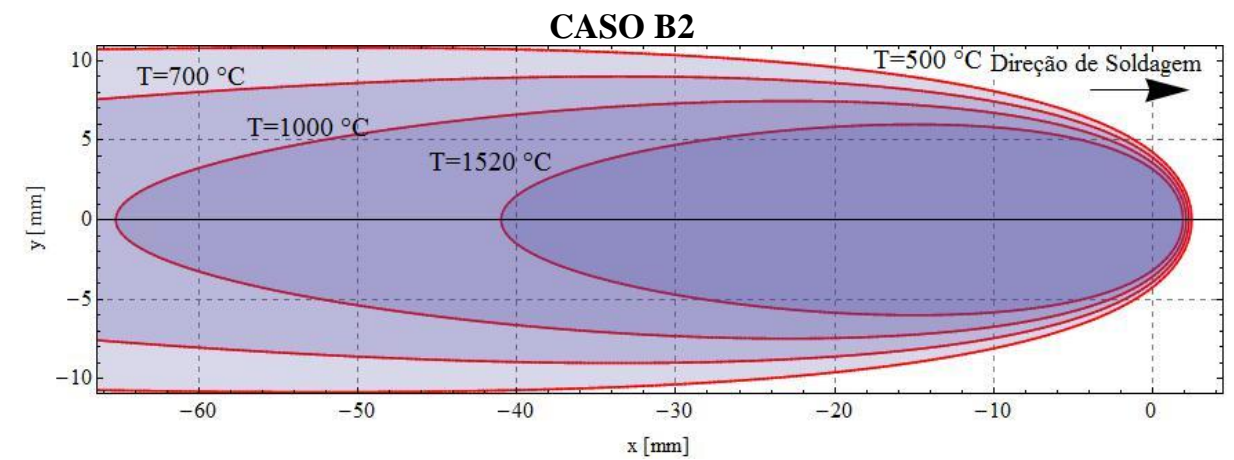

(b)

Figura (5b). Formatos dos contornos das isotermas do caso B2, Corrente $=480 \mathrm{~A}$ e a velocidade de soldagem $v=8 \mathrm{~mm} / \mathrm{s}$. 
As Figs. (5a) e (5b) apresentam os resultados obtidos dos parâmetros para um aço carbono, variando-se as velocidades de soldagem e a corrente de soldagem. Com isso percebe-se um acentuado prolongamento no formato das isotermas em relação à direção de soldagem, o mesmo comportamento observado nas Figs. (4a) e (4b). No entanto, sob o ponto de vista das propriedades dos materiais, a difusividade térmica influencia no prolongamento dos contornos, o aço possui baixa difusividade comparado ao alumínio e, com isso, a modificação dos formatos das isotermas é bem mais acentuado quando comparados aos do alumínio.

Os códigos implementados utilizando os três métodos numéricos, foram submetidos à simulação para os casos apresentados na Tab. (1). A estimativa inicial foi a mesma para os três métodos, assim como a mesma precisão de erro e o número de pontos na primeira rodada. O método de melhor desempenho foi o da bisseção para o caso A1 e A2 e para o caso B1 e B2 a secante se destacou, como mostra a Tab. (2), ou seja, para materiais com alta difusividade térmica, variando-se a velocidade de soldagem e a corrente, a bisseção é recomendável. No entanto, para materiais com baixo valores de difusividade, a secante se comportaria melhor em relação à bisseção. O método de, Newton-Raphson não convergiu, devido ao distanciamento ao longo das iterações, a partir da estimativa inicial fixa.

Tabela 2. Resultados obtidos após a primeira simulação para obtenção do contorno da isoterma, utilizando a estimativa inicial fixa.

\begin{tabular}{|c|l|c|c|c|}
\hline \multirow{2}{*}{ Casos } & \multicolumn{1}{|c|}{ Medidas } & \multicolumn{3}{c|}{ Métodos Numéricos } \\
\cline { 2 - 5 } & & Bisseção & Newton-Raphson & Secante \\
\hline \multirow{3}{*}{ A1 } & Tempo Computacional (s) & 1.0150 & Não Convergiu & 1.0290 \\
\cline { 2 - 5 } & Média de Iterações & 49.3500 & Não Convergiu & 23.4800 \\
\cline { 2 - 5 } & Desvio Padrão & 14.3620 & Não Convergiu & 27.7390 \\
\hline \multirow{3}{*}{ A2 } & Tempo Computacional (s) & 4.1190 & Não Convergiu & 4.6810 \\
\cline { 2 - 5 } & Média de Iterações & 28.8570 & Não Convergiu & 35.7070 \\
\cline { 2 - 5 } & Desvio Padrão & 10.6270 & Não Convergiu & 52.9850 \\
\hline \multirow{3}{*}{ B1 } & Tempo Computacional (s) & 154.1749 & Não Convergiu & 82.5080 \\
\cline { 2 - 5 } & Média de Iterações & 5.5599 & Não Convergiu & 65.6620 \\
\cline { 2 - 5 } & Desvio Padrão & 4.1268 & Não Convergiu & 146.7300 \\
\hline \multirow{3}{*}{ B2 } & Tempo Computacional (s) & 1052.3360 & Não Convergiu & 369.3010 \\
\cline { 2 - 5 } & Média de Iterações & 1.1765 & Não Convergiu & 78.9330 \\
\cline { 2 - 5 } & Desvio Padrão & 1.6865 & Não Convergiu & 199.1200 \\
\hline
\end{tabular}

Por tratar-se de uma solução com múltiplas raízes (pontos), percebeu-se que os resultados preliminares acima apresentados ocorreram devido à estimativa inicial fixa ao longo da determinação das raízes, como também a variação da quantidade de iterações para solução de cada caso, mesmo ocorrendo convergência na bisseção e secante.

Diante disso, ficou claro que, ao tornar a estimativa inicial da próxima raiz, a raiz anterior, ou seja, tornar a estimativa inicial dinâmica, haveria uma aproximação maior da próxima raiz a ser determinada, otimizando o processo iterativo e consequentemente o desempenho do código. Na Tab. (3) são apresentados os resultados obtidos após implementação da rotina que torna a raiz calculada como estimativa inicial da próxima raiz, os três métodos responderam a esta ação de forma mais efetiva, comportando-se com maior estabilidade e menor custo computacional.

Tabela 3. Resultados obtidos após a implementação da otimização da estimativa inicial.

\begin{tabular}{|c|l|c|c|c|}
\hline \multirow{2}{*}{ Casos } & \multicolumn{1}{|c|}{ Medidas } & \multicolumn{3}{c|}{ Métodos Numéricos } \\
\cline { 3 - 5 } & & Bisseção & Newton-Raphson & Secante \\
\hline \multirow{3}{*}{ A1 } & Tempo Computacional (s) & 0.9059 & 0.2960 & 0.3739 \\
\cline { 2 - 5 } & Média de Iterações & 48.8300 & 2.4300 & 3.0100 \\
\cline { 2 - 5 } & Desvio Padrão & 17.1490 & 0.6237 & 1.7494 \\
\hline \multirow{3}{*}{ A2 } & Tempo Computacional (s) & 2.1990 & 0.5450 & 0.8419 \\
\cline { 2 - 5 } & Média de Iterações & 27.4512 & 2.2533 & 2.5382 \\
\cline { 2 - 5 } & Desvio Padrão & 11.1248 & 0.4761 & 1.7848 \\
\hline \multirow{3}{*}{ B1 } & Tempo Computacional (s) & 30.9180 & 2.8700 & 7.4549 \\
\cline { 2 - 5 } & Média de Iterações & 5.4361 & 2.0345 & 2.1486 \\
\cline { 2 - 5 } & Desvio Padrão & 4.4579 & 0.2048 & 2.3939 \\
\hline \multirow{3}{*}{ B2 } & Tempo Computacional (s) & 122.7869 & 9.5480 & 27.1289 \\
\cline { 2 - 5 } & Média de Iterações & 2.0120 & 2.0104 & 2.0599 \\
\cline { 2 - 5 } & Desvio Padrão & 0.1124 & 2.5603 \\
\hline
\end{tabular}

Há de se notar a expressiva vantagem do método de Newton-Raphson em relação aos outros dois métodos, mesmo necessitando do calculo da derivada da função, o método obteve menor tempo computacional e maior estabilidade, tendo como base o baixo desvio padrão da média de iterações para os casos simulados. 


\section{CONCLUSÕES}

Com base nos resultados e discussões, concluiu-se que o método de Newton-Raphson é formalmente o menos dispendioso computacionalmente, e mais estável para o estudo de caso apresentado neste trabalho, quando utilizada a otimização da estimativa inicial, pois na primeira simulação não houve convergência deste método, diante disso as seguintes conclusões podem ser observadas:

- A melhoria dos resultados preliminares pode ser obtida a partir da inclusão no código, a rotina que torna dinâmica a estimativa inicial da próxima raiz;

- Os resultados obtidos na simulação para os casos estudados estão coerentes com o conteúdo encontrado na literatura, conforme (Vetter, Santos e Marques ,2006; Cota, Bastos e Ferreira, 2012).

- Diante dos resultados apresentados, pode-se prever o comportamento do contorno da isoterma e então ajustar os parâmetros de soldagem para o tipo de material a ser soldado, minimizando a ocorrência de erros e retrabalhos na indústria.

\section{AGRADECIMENTOS}

Os autores gostariam de agradecer ao Programa de Pós-Graduação em Engenharia Metalúrgica e de Materiais da USP - Universidade de São Paulo, IFES - Instituto Federal de Educação, Ciência e Tecnologia do Espírito Santo e a FAPEAM- Fundação de Amparo à Pesquisa do Estado do Amazonas pelo apoio financeiro.

\section{REFERÊNCIAS}

Biswa, N. D. 2011. "Department of Mathematical Sciences." Biswa Nath Datta. http://www.math.niu.edu/ dattab/MATH435.2013/ROOT_FINDING.pdf (acesso em 3 de junho de 2014).

Cota, E. F., Bastos, A. F. , Silva, Pereira, H. A. 2012. "Aplicação do Método de Newton-Raphson no Controle da Resistência Externa de Geradores Eólicos Durante Afundamentos de Tensão." Simpósio Brasileiro de Sistemas Elétricos, vol. 1. Goiânia, Goiás.

Ehiwario, J.C., Aghamie, S.O. 2014. "Comparative Study of Bisection, Newton-Raphson and Secant Methods of RootFinding Problems.” IOSR Journal of Engineering (IOSRJEN), vol. 4 (pp. 01-07)

Grong, O., 1997. Metallurgical Modelling of Welding. Trondhein, Norway: Ed. The University Press Cambridge, 677 p.

Guimarães, P.B., Moura, M. de L. M., Silva, da F. J. R., Fernandes, E. L., Nascimento, do S. C. A., Barbosa, A. M. J., Ferreira, S. A. R. 2012. "Obtenção Numérica Dos Ciclos Térmicos De Soldagem Do Aço Inoxidável AISI 304." VII Congresso Nacional de Engenharia Mecânica. Maranhão, Brasil.

Miranda, J.H. et al. 2006. "Aplicação de métodos numéricos para estimativa de variáveis psicrométricas." Scielo Brasil, Engenharia Agrícola, vol. 3 No. 3, 686-694.

Myhr, O. R., e O. Grong. "Dimensionless maps for heat flow analyses in fusion welding." Acta metallurgica et materialia, 1990: 449-460.

Rosenthal, D. 1941. Mathematical Theory of Heat Distribution during Welding and Cutting. Welding Journal, 20(5), 220-234.

Vetter, V. N., Santos, S. P. G., Marques, P. R. 2006. "Método da Secante Para Resolução de equações do tipo f(x)=0." O SEGeT - Simpósio de Excelência em Gestão e Tecnologia, vol. 1, (pp. 1-9). Rio de Janeiro, Brasil.

Wainer, Emílio, Sérgio Duarte Brandi, e Fábio Decourt Homem Mello,1992. Soldagem: Processo e Metalurgia. São Paulo, Brasil: Edgard Blücher Ltda. 494p.

\section{RESPONSABILIDADE AUTORAL}

Os autores são os únicos responsáveis pelo conteúdo do material impresso incluído neste trabalho. 


\title{
COMPARATIVE STUDY OF CONVERGENCE OF NUMERICAL METHODS ITERATIVE APPLIED IN SOLUTION OF EQUATION HEAT TRANSFER PROPOSAL BY ROSENTHAL UNDER VARIATION PARAMETERS OF WELDING.
}

\author{
Antonio do Nascimento Silva Alves, ansa@usp.br ${ }^{1}$ \\ Rubelmar Maia de Azevedo Cruz Neto, rubelmar.neto@ usp.br ${ }^{1}$ \\ Sergio Duarte Brandi, sebrandi@ usp.br ${ }^{1}$ \\ Dario Magno Batista Ferreira, dariomagno@ usp.br ${ }^{1}$
}

${ }^{1}$ USP - Dep. de Eng. Metalúrgica e de Materiais. Av. Professor Mello Moraes, 2463, CEP 05508-030, São Paulo - SP

\begin{abstract}
Roots evaluation or function zero of transcendental equations in reduced form depends on the behavior of these equations near to the roots, such as they need reliable numerical methods with low computational cost for their most effective evaluation. These methods are widely used in engineering. The geometry of the isotherms in welded joints of metal sheets are of fundamental importance to determine their mechanical properties, microstructure formation and cooling rate. To reach this purpose it is used the heat transfer equation solution proposed by Rosenthal, considering only the effect of conduction as the heat transfer mode, neglecting the contributions of convection and radiation. Varying the welding parameters, namely, current, voltage, welding speed and process efficiency, generates as consequence different isotherm geometries. To evaluate the solution equation above mentioned, we used iterative numerical methods. This paper presents a comparative stability study about convergence of Bisection, NewtonRaphson's and Secant methods, in terms of iterations number, computational time and accuracy of the numerical error during obtaining roots or function zeros. Under these conditions, the best convergence was Newton-Raphson method with fewer iterations and less computational time than other ones.
\end{abstract}

Keywords: Heat transfer in welding, Numerical Methods, Contour isotherms, Computational time, Rosenthal equation. 